\title{
Study of the Tow Buckling Defect During the Shaping of Structural Composites Based on Synthetic and Vegetal Fibres
}

\section{Étude des Défauts de Bouclage Lors de la Mise en Forme de Composites Structuraux à Base de Fibres Synthétiques et Végétales}

\author{
Mohamed M. Salem*, Emmanuel De Luycker, Marina Fazzini, Pierre Ouagne \\ Laboratoire Génie de Production (LGP), INP-ENIT, Univ. de Toulouse. 47, avenue d'Azereix - BP 1629 - 65016 Tarbes \\ CEDEX, France
}

Corresponding Author Email: mohamed-medhat.salem@enit.fr

https://doi.org/10.18280/rcma.290506

Received: 8 March 2018

Accepted: 26 January 2019

\section{Keywords: \\ composite manufacturing, preforming defects, textile reinforcement, tow buckling, full field strain measurement}

\section{Mots-clés :}

fabrication de composites, défauts de préformage, renfort textile, bouclage de mèches, mesure de champ

\begin{abstract}
:
During the complex shape forming of composite fibrous reinforcement, the in-plane bending of roving tows results in an out-of-plane deflection, along with a rotation on its central axis. The need to accurately follow and quantify the formation mechanism of such defect has led us to consider two 3D imaging techniques which were tested and compared in this work. Solutions to prevent the appearance of this defect were proposed with success.
\end{abstract}

\section{RÉSUMÉ :}

Pendant la mise en forme complexe de composites à renfort fibreux, une flexion dans le plan des mèches plates entraine une déflexion hors plan accompagnée d'une rotation autour de leur ligne moyenne. La nécessité de comprendre et de quantifier avec précision le mécanisme de formation d'un tel défaut nous a conduit à considérer des techniques d'imagerie 3D qui ont été testées et comparées dans ce travail. Des solutions pour prévenir l'apparition de ce défaut ont été proposées avec succès.

\section{INTRODUCTION}

It is no surprise, considering the attention that the structural composite materials are getting lately in recent works, that they present interesting mechanical properties. Coupled with ecological challenges such as waste and gas emission reduction by prioritizing, a recyclable approach and reducing weight to reduce energy consumption justify the choice of composite materials with natural fibers to replace the metallic ones.

To manufacture said composite parts, shaping techniques, such as Thermoplastic-Matrix Composites Stamping and Resin Transfer Molding have been used. Yet these techniques are far from flawless and result in various defects, some of which have been investigated [1-3] and others such as the tow buckling that remains to be fully understood.

In this work, the tow buckling defect was investigated. The buckles appear on woven reinforcement during the complex shape forming. To fully grasp the kinematic involved in the development of the defect, an experimental buckling device was designed in order to reproduce this defect in situ [5]. A non-intrusive 3D measurement technique was also required, but regarding the peculiar nature of the reinforcement, the most suitable candidate had to be determined between different optical measurement systems.

The optical systems that were chosen and compared were the Stereo-Digital image Correlation (S-DIC), the Fringes projection and the Variable focus microscopy. For each system, the reinforcement needed a pre-treatment to get rid of surface stray fibres that causes data noise and even data loss. The same conditions were recreated for each technique and the slope of the buckle during different stages of buckling was compared. The comparison between the techniques yielded similar results and the S-DIC system was used for added accessible data (point by point displacement) and convenience.

Buckling apparition criteria and their influence on the development of the buckles were then investigated using the buckling device and different reinforcement samples. The data collected will be used to analyse and comprehend the buckling's kinematic and will be later used to compare with theoretical results obtained by an analytical model that is being currently investigated and developed.

Soixante-dix pour cent des pièces structurelles et semistructurelles utilisées dans l'industrie automobile sont métalliques. Une double approche écologique (réduction des émissions de $\mathrm{CO}_{2}$ en réduisant le poids des pièces et en améliorant la recyclabilité des pièces) justifie le choix de matériaux composites avec des renforts bidirectionnels en fibres naturelles pour remplacer les matériaux métalliques actuellement utilisés.

La faisabilité de fabrication de pièces structurelles de géométries complexes et spécifiques, sans défaut, via des processus combinant, un taux de production élevé, un faible coût et une forme accessible, comme le procédé d'emboutissage de renforts, est un défi scientifique majeur. Cette faisabilité dépend non seulement des géométries des formes à réaliser, mais aussi des architectures du renfort textile aux échelles macroscopique et mésoscopique.

Si certains défauts, tels que les plis, ont déjà fait l'objet d'études approfondies [1-3], ce n'est pas nécessairement le cas 
pour les défauts de bouclage et de glissement de mèches qui peuvent apparaître lors de la mise en forme des textiles techniques. Ces défauts conduisent à l'apparition de lacunes et de discontinuités dans la structure du renfort fibreux $[4,5]$.

Cet article propose d'étudier le défaut de bouclage et sa détection avec différents systèmes optiques tels que la StéréoCorrélation d'Images Numérique (S-DIC), la microscopie à focale variable et la projection de franges. Alors que les deux dernières techniques permettent de reconstruire un profil avec une très bonne résolution et précision, la première permet, en plus, d'accéder au déplacement et à la déformation subis en chaque point de la surface reconstruite. Le défaut de bouclage est étudié indépendamment du procédé de mise en forme avec un dispositif spécialement conçu pour recréer les conditions d'apparition. Le montage expérimental permettra de réaliser une analyse cinématique multi-échelle du phénomène de bouclage, en estimant la hauteur et l'élévation du profil de la boucle et les déformations subies par les fibres.

\section{MONTAGE EXPÉRIMENTAL}

\subsection{Dispositif de bouclage des mèches}

Le défaut de bouclage est principalement le résultat d'une combinaison entre la flexion des mèches plates dans leur plan et le niveau de tension appliqué aux mèches perpendiculaires à celles montrant des boucles [4]. Un dispositif a donc été conçu pour imposer séparément des angles de flexion dans le plan des mèches transversales et des tensions variables dans les mèches longitudinales. La conception de la partie mécanique du dispositif a été décrite en détail dans [5] et est résumée dans la Figure 1.
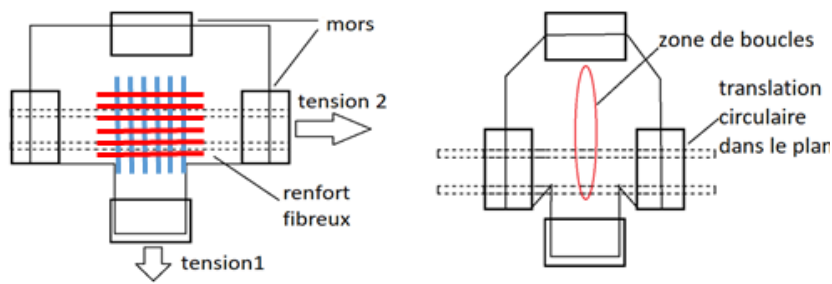

(a)

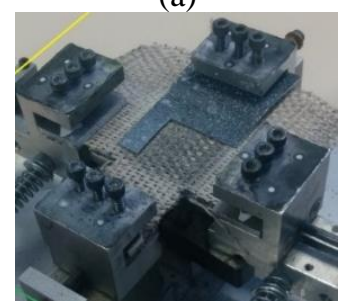

(c)

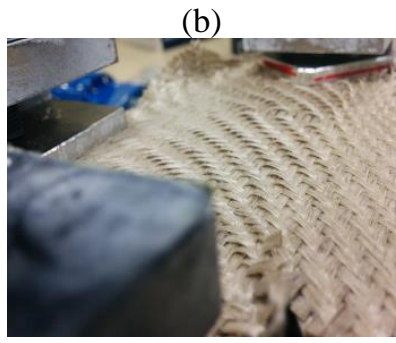

(d)

Figure 1. Principe du dispositif de bouclage des mèches avec (a) et (b) des vues schématiques et (c) et (d) des vues en cours d'essai

Le dispositif a été instrumenté des capteurs de charge et d'un système S-DIC. La géométrie des échantillons est adaptée pour maximiser la zone de bouclage. Les échantillons sont maintenus par des mors dans deux directions sur lesquelles différents niveaux de tension peuvent être imposés.

Les mors qui permettent d'appliquer la charge 2 dans le réseau de mèches transversales (réseau sur lequel les boucles apparaissent) subissent une translation circulaire dans le plan comme illustré par la Figure 1 (b). Les mors imposent aux mèches un angle de flexion qui peut être calculé à partir de la distance relative des mors par rapport à leur distance initiale comme illustré sur la Figure 2 (b).

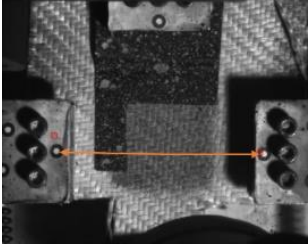

(a)

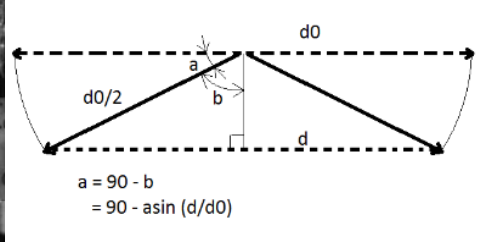

(b)
Figure 2. Calcul de l'angle de flexion dans le plan des mèches avec (a) vue en cours d'essai et (b) schéma de la mesure

\subsection{Systèmes de mesure}

\subsubsection{Stéréo-Corrélation d'Image Numériques(S-DIC)}

Le système de stéréo-corrélation utilisé est composé de deux caméras numériques capables de capturer douze images par seconde associées au logiciel GOM ARAMIS ${ }^{\circledR}$. La stéréo corrélation est une méthode de mesure de champs qui combine la corrélation d'images numériques (DIC) et la vision stéréoscopique.

Le DIC permet de déterminer le champ de déplacements plan d'un objet en enregistrant une image à chaque état de déformation de ce dernier. Deux images correspondant à deux états de déformation d'un objet sont utilisées pour déterminer le correspondant d'un point et sa signature à partir de la première image dans la seconde. Pour cela, une fonction de similitude est utilisée. En pratique, une seule valeur ne correspond pas à la signature unique d'un point, d'où l'utilisation de pixels voisins. L'appariement d'images acquises par une seule caméra, à des moments différents, sur un objet qui s'est déformé, est appelé appariement temporel, ou suivi. De son principe, la technique de corrélation ne peut fonctionner correctement qu'avec des objets ayant une surface avec une texture suffisamment aléatoire. Si l'objet n'est pas texturé naturellement ou si sa texture n'est pas suffisamment discriminante, différentes techniques existent pour permettre l'utilisation de la corrélation (mouchetis par projection de peinture).

La vision stéréoscopique est basée sur le principe que l'information en profondeur (3D) qui peut être obtenue par triangulation à partir de deux images ayant une partie commune dans leur champ de vision. L'obtention de ces données implique l'étalonnage $\mathrm{du}$ capteur de vision stéréoscopique. L'étalonnage d'une caméra consiste à déterminer ses paramètres intrinsèques. L'étalonnage d'un capteur de vision stéréoscopique composé de deux caméras dépend de la détermination des paramètres intrinsèques de chaque caméra, ainsi que de la position et de l'orientation relative des deux caméras. Ces paramètres sont nécessaires pour calculer les coordonnées tridimensionnelles d'un groupe de pixels, correspondant à un seul point, appariés dans les images des deux capteurs. L'appariement de deux images acquises, à un instant donné, par deux caméras stéréoscopiques dépendantes est appelé appariement par stéréo-corrélation.

La mesure de champs de déplacement 3D à l'aide de la vision stéréoscopique met en œuvre simultanément une technique d'appariement temporel et un couplage stéréo- 
corrélation. La technique de corrélation peut également être utilisée pour rechercher des correspondants stéréoscopiques à partir d'une paire d'images stéréoscopiques. Notons qu'une différence essentielle distingue l'appariement temporel par corrélation de l'appariement par stéréo-corrélation : dans le cas de la vision stéréoscopique, il existe une contrainte géométrique dite épipolaire qui peut guider la recherche du stéréo-correspondant.

\subsubsection{Microscopie à focale variable}

Le microscope utilisé, Infinite Focus, Alicona ${ }^{\circledR}$ est un système de mesure de la topographie qui utilise le principe de la variation du foyer. Ce système capture les images de la surface à mesurer en faisant varier la distance focale. Grâce à un algorithme de détection de contour, l'image correspondant au meilleur foyer est sélectionnée pour chaque point de la surface. Il est alors possible de connecter l'image à la distance focale et donc au relief de la surface.

\subsubsection{Projection de franges}

La projection de franges est une technique de lumière structurée où une grille de franges noires et blanches alternées est projetée sur un objet. La distance entre les franges est connue. La grille maintenant déformée est captée par un ou plusieurs capteurs optiques. Chaque point de la grille est triangulé en fonction d'un étalonnage effectué avant la mesure. L'étalonnage est effectué en projetant une grille sur une plaque référencée. La projection de frange a été réalisée à l'aide du système GOM ATOS $\AA$

\section{RESULTATS}

Une comparaison initiale entre les différentes techniques optiques a été réalisée sur un renfort sergé de lin encollé représenté sur la Figure 3.

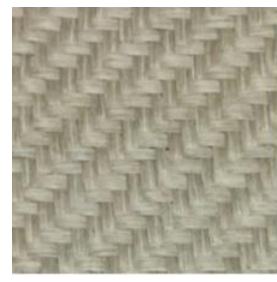

Figure 3. Renfort sergé $2 \times 2$ en lin encollé

En tenant compte de la nature particulière du renfort, deux problèmes majeurs ont dû être surmontés pour utiliser pleinement la stéréo-corrélation. Le premier problème était l'abondance des fibres et des extrémités des fibres qui sortent du renfort de lin. Ces fibres sont si fines qu'elles semblent se fondre dans le plan du renfort alors qu'elles sont à plusieurs millimètres hors du plan. Cela a conduit à plusieurs décorrélations sur la surface et a nécessité le rasage du renfort. Le deuxième problème était dû à la surface trop réfléchissante des fibres de lin. Une fine couche de poudre révélatrice blanche a été appliquée pour diminuer la réflexion et un fin mouchetis noir a été appliqué sur le dessus à l'aide d'une bombe de peinture pour augmenter le contraste nécessaire à la corrélation.

Au cours des essais de bouclage, la flexion dans le plan des mèches entraîne un déplacement des mèches transversales, tandis que les mèches longitudinales restent immobiles. Les mèches se chevauchent et couvrent des parties des mouchetis préexistants tout en donnant l'apparence de zones sans mouchetis. Cela entraîne finalement la perte d'informations corrélées au fil du temps.

La microscopie à variation de focale ainsi que la projection de franges éliminent le besoin d'un mouchetis. La surface transcrite (cf. Figure 4 (a)) est beaucoup plus précise avec 100 $\mathrm{nm}$ de résolution verticale pour le microscope à variation focale contre $10 \mu \mathrm{m}$ pour la projection de franges (cf. Figure 4 (b)) et $30 \mu \mathrm{m}$ pour la stéréo-corrélation (cf. Figure 4 (c)).

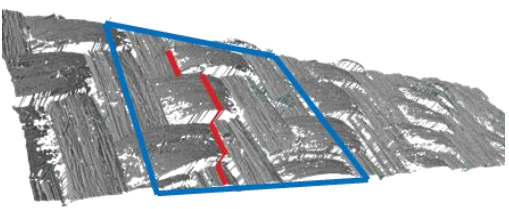

(a)

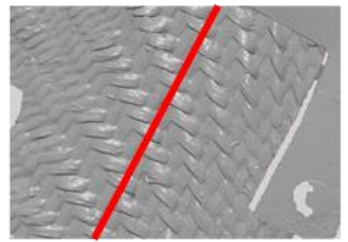

(b)

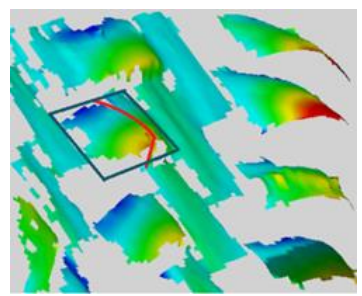

(c)

Figure 4. Surface de renfort reconstruite par a) microscopie à focale variable, b) projection de franges et c) stéréocorrélation

Le microscope offre une grande précision dans la reproduction de la surface, comme indiqué précédemment sur la Figure 4 (a). La forme de la section est clairement définie et offre une localisation précise en $3 \mathrm{D}$ de chaque point. Pourtant, lier une étape à la suivante est une tâche plus ardue. Compte tenu du fait que le microscope n'a pas la capacité de suivre le déplacement de la mèche (flambement couplé à la rotation impliqués dans la formation de la boucle), il est extrêmement difficile d'extraire le déplacement des courbes de données acquises alignées par défaut dans les graphes par la méthode du meilleur ajustement, comme indiqué sur la Figure 5, ce qui a pour effet de minimiser le déplacement dans certains cas (cf. Figure 5). La projection de franges a également une précision élevée avec l'avantage d'une acquisition beaucoup plus rapide. Mais, la technique souffre également du même inconvénient que la technique précédente, étant incapable de donner des informations sur le déplacement et les graphes ont dû être alignés manuellement comme montré sur la Figure 6. Le dispositif de stéréo-corrélation nous fournit à la fois la forme et le déplacement de chaque point corrélé comme le montrent les Figures 7 (a) et 7 (b). Tandis que la forme de la mèche est intéressante, puisque les premières expérimentations suggèrent que la disposition initiale de la mèche semble influencer l'élévation maximale finale, le déplacement est crucial dans une conception expérimentale future pour pouvoir prédire la formation d'une boucle. 


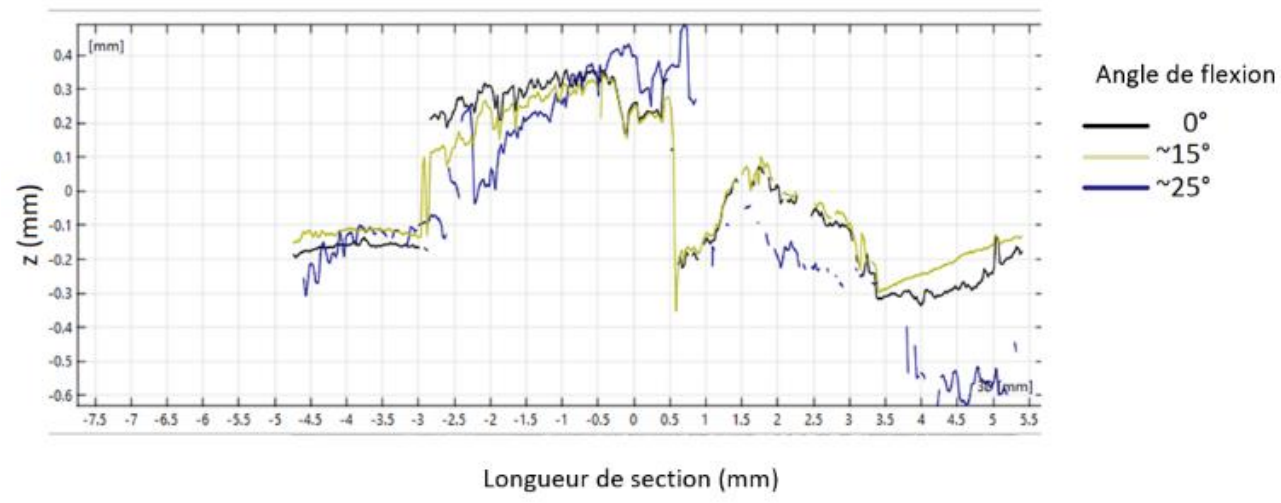

Figure 5. Profil d'une mèche de lin encollé sergé $2 \times 2$ obtenue par microscopie à focale variable

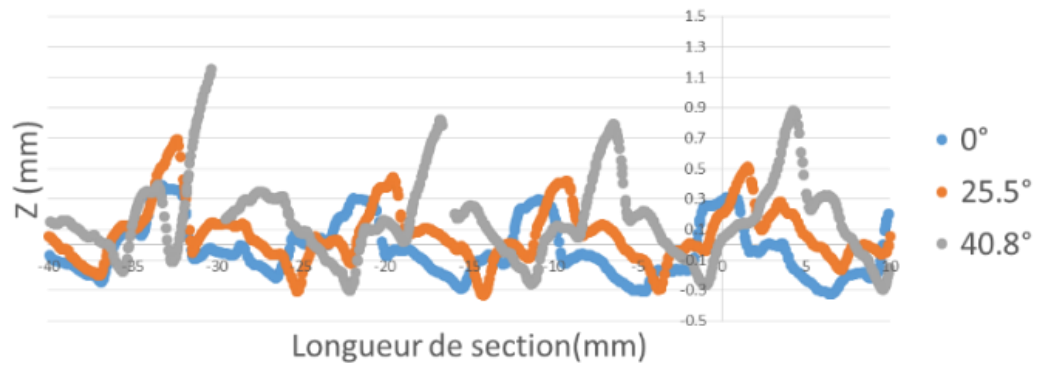

Figure 6. Profil d'une mèche de lin encollé sergé $2 \times 2$ obtenue par projection de franges

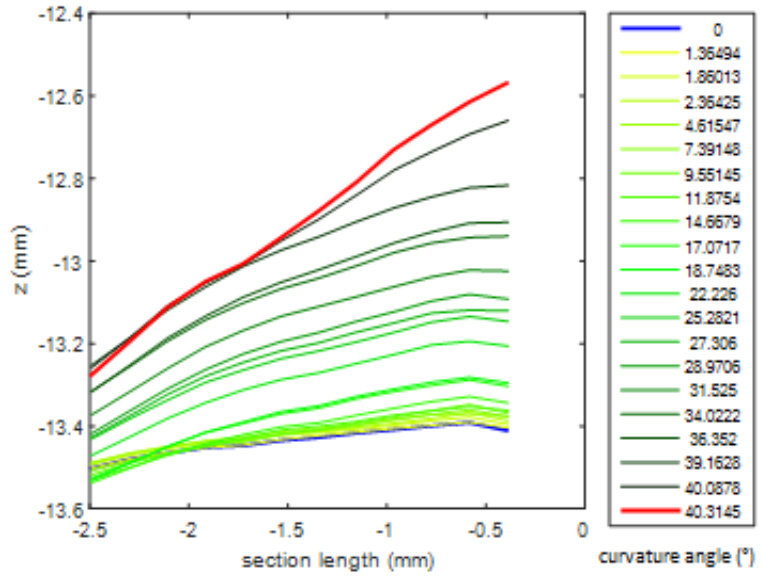

(a)

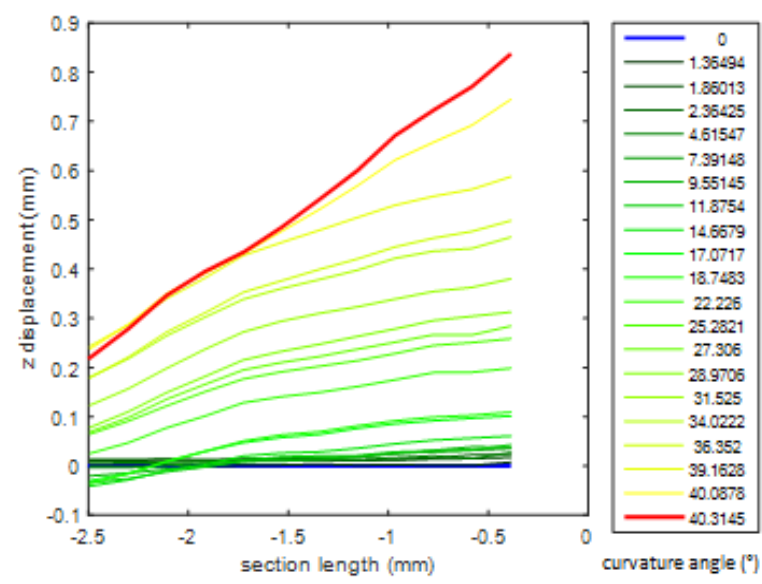

(b)

Figure 7. Profil d'une mèche de lin encollé sergé $2 \times 2$ obtenu par S-DIC pour différents angles de flexion (a) et déplacements hors-plan (d)
Cependant, même avec les lacunes des différentes techniques, la comparaison des profils entre eux reste assez intéressante. En regroupant les résultats des Figures 5, 6 et 7 sur un même graphe et en prenant comme référence commune la position du réseau longitudinal qui ne boucle pas, nous pouvons avoir une idée du déplacement et de la rotation de la boucle. Les résultats des trois configurations sont présentés dans la Figure 8 et résumés dans le Tableau 1.

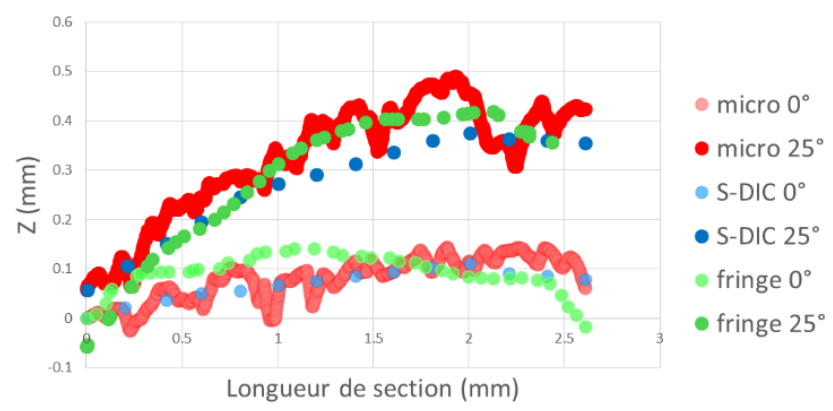

Figure 8. Superposition des profils de mèches obtenus par microscopie à focale variable, projection de franges et Stéréo-Corrélation pour des angles de flexion de $0^{\circ}$ et $25^{\circ}$

Tableau 1. Élévation et rotation maximales mesurées par stéréo-corrélation, microscopie à focale variable et projection de franges pour un renfort sergé $2 \times 2$ de lin encollé avec un angle de flexion de $20^{\circ}$

\begin{tabular}{ccc}
\hline \multicolumn{3}{c}{ Élévation maximale (mm) } \\
\hline S-DIC & Microscopie & franges \\
\hline 0.38 & 0.45 & 0.41 \\
\hline \multicolumn{3}{c}{ Rotation $\left(^{\circ}\right)$} \\
\hline S-DIC & Microscopie & franges \\
\hline 5.24 & 6.51 & 6.27 \\
\hline
\end{tabular}


Les valeurs enregistrées avec les trois techniques sont assez similaires et du même ordre de grandeur avec quelques petites différences qui étaient attendues, considérant que les mêmes conditions expérimentales ne pouvaient pas être reproduites exactement avec les trois dispositifs. Cela nous permet toujours de valider les résultats obtenus à partir du S-DIC.

La Figure 9 représente le tracé de l'élévation maximale (a) et de l'angle de rotation (b) de la mèche par rapport à l'angle de courbure pour trois renforts différents, énumérés sur le Tableau 2. Les renforts ont été soumis à une charge de $300 \mathrm{~N}$ dans le réseau de mèches fixes et de $20 \mathrm{~N}$ dans le réseau fléchi avec un angle de $40^{\circ}$. Comme le montre la Figure 9, on obtient une idée de la cinématique impliquée lors de la formation de la boucle ainsi que l'influence de la contexture du renfort. Plus la rigidité à la flexion est élevée, plus les boucles se manifestent tôt et flambent haut.

Tenant compte de la variation de la longueur et de la largeur libre des mèches et en utilisant les mêmes conditions expérimentales que précédemment, nous avons comparé certains renforts en sergé $2 \times 2$ de lin désencollé sur la Figure 8. Comme la longueur libre augmente comme le montre la Figure 10 (a), le flambement et la rotation de la mèche augmentent aussi. Ceci est expliqué par la liberté supplémentaire pour la boucle de flamber et de se développer. Mais à mesure que la largeur non supportée augmente comme le montre la Figure 10 .

Tableau 2. Caractéristiques des renforts utilisés dans les tests de bouclage

\begin{tabular}{|c|c|c|c|c|c|c|c|}
\hline Armure & matériau & $\begin{array}{c}\text { Masse } \\
\text { surfacique } \\
\left(\mathrm{g} / \mathbf{m}^{2}\right)\end{array}$ & $\begin{array}{l}\text { Densité } \\
\text { chaine } \\
\left(\mathbf{m}^{-1}\right)\end{array}$ & $\begin{array}{c}\text { Densité } \\
\text { trame }\left(\mathbf{m}^{-1}\right)\end{array}$ & $\begin{array}{l}\text { Longueur libre } \\
\text { de mèche (mm) }\end{array}$ & $\begin{array}{c}\text { Largeur libre } \\
\text { de mèche } \\
(\mathrm{mm})\end{array}$ & $\begin{array}{c}\text { Rigidité en } \\
\text { flexion de } \\
\text { mèche (N.mm) }\end{array}$ \\
\hline Sergé 2x2 & Lin encollé & 476 & 380 & 385 & 6.11 & 2.48 & 1.18 \\
\hline Sergé 2x2 & Lin désencollé & 465 & 380 & 430 & 5.66 & 2.40 & 0.72 \\
\hline Sergé 2x2 & $\begin{array}{l}\text { Fibre de } \\
\text { carbone }\end{array}$ & 600 & 380 & 380 & 6.42 & 2.32 & 7.55 \\
\hline
\end{tabular}

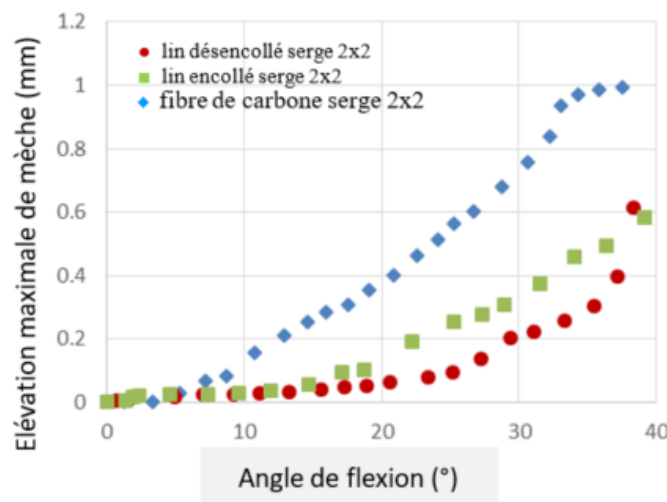

(a)

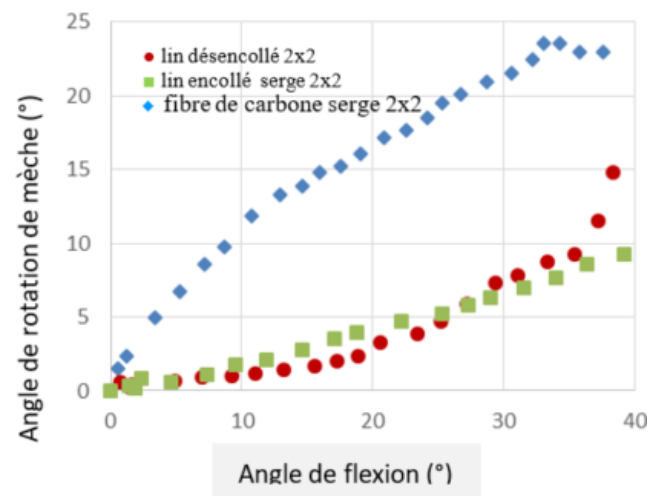

(b)

Figure 9. Élévation maximale (a) et angle de rotation de la mèche (b) en fonction de l'angle de flexion

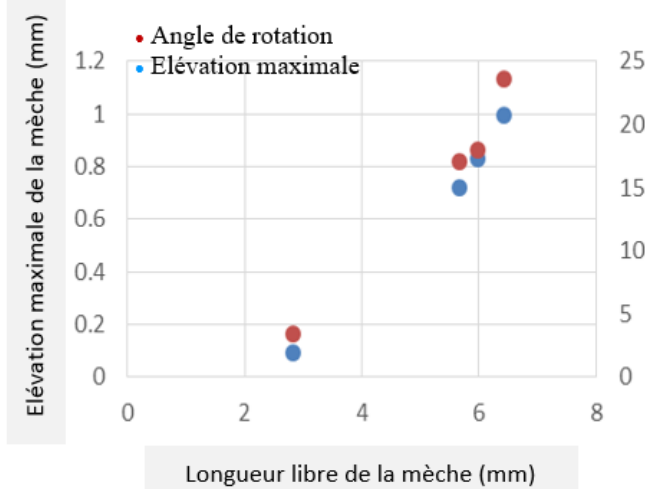

(a)

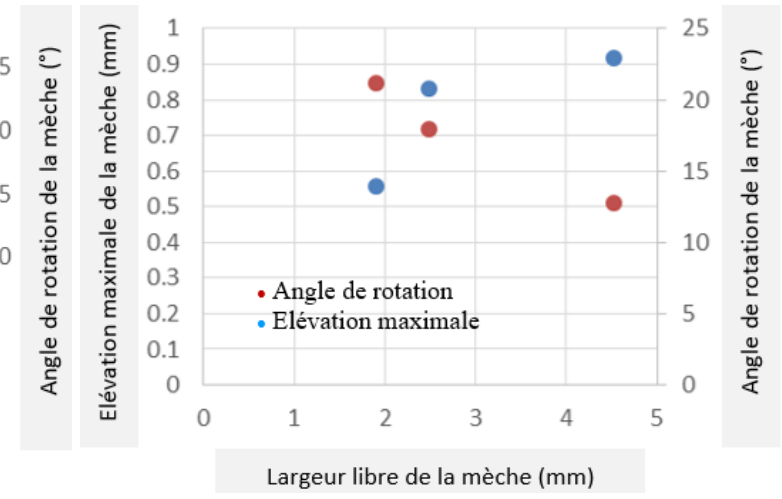

(b)

Figure 10. Élévation maximale et angle de rotation de la mèche en fonction de la longueur libre de la mèche (a) et en fonction de la largeur libre de la mèche (b)

(b), le flambement augmente tandis que la rotation diminue. Cela pourrait s'expliquer par de plus petites boucles relativement à la largeur des mèches, ce qui signifie que, même si elles s'élèvent plus haut dans l'absolu, leur rotation diminue.

\section{CONCLUSION}

Le bouclage des mèches a été étudié en détail dans cette étude par différentes approches. Différents systèmes de mesure ont été testés pour étudier les paramètres contrôlant l'apparition du défaut. Les valeurs expérimentales enregistrées 
par S-DIC, projection de franges et microscopie à variation focale sont assez similaires et du même ordre de grandeur. De petites différences étaient attendues pour des raisons de reproductibilité expérimentale de l'essai pour les trois échantillons testés.

Les effets de certains paramètres ont été étudiés et montrent que les boucles dépendent fortement de la rigidité de flexion, de la longueur et de la largeur libre des mèches. D'autres paramètres sont également en cours d'étude et un modèle analytique est en cours de mise en place pour confirmer les résultats expérimentaux.

\section{REFERENCES}

[1] Gereke. T., Döbrich. O., Hübner. M., Cherif. C., (2013). Experimental and computational composite textile reinforcement forming: A review. Composites Part A, 46: $1-10$. http://dx.doi.org/10.1016/j.compositesa.2012.10.004

[2] Bloom, L.D., Wang, J., Potter, K.D. (2013). Damage progression and defect sensitivity: an experimental study of representative wrinkles in tension. Composites Part B, 45:

449-458.

http://dx.doi.org/10.1016/j.compositesb.2012.05.021

[3] Lee, J., Hong, S., Yu, W., Kang, T. (2007). The effect of blank holder force on the stamp forming behavior of noncrimp fabric with a chain stitch. Compos. Sci. Technol., 67:

357-366. http://dx.doi.org/10.1016/j.compscitech.2006.09.009

[4] Ouagne, P., Soulat, D., Hivet, G., Allaoui, S., Duriatti, D., (2011). Analysis of defects during the preforming of a woven flax. Adv. Compos. Lett., 20: 105-108. http://dx.doi.org/10.1177/096369351102000403

[5] Tephany, C., Gillibert, J., Ouagne, P., Hivet, G., Allaoui, S., Soulat, D. (2016). Development of an experimental bench to reproduce the tow buckling defect appearing during the complex shape forming of structural flax based woven composite reinforcements. Composites Part A, 81 :

22-33. 